\title{
WEYL'S THEOREM, TENSOR PRODUCTS AND MULTIPLICATION OPERATORS II
}

\author{
BHAGGY DUGGAL \\ 8 Redwood Grove, Northfield Avenue, Ealing, London W5 4SZ, UK \\ e-mail:bpduggal@yahoo.co.uk \\ ROBIN HARTE \\ School of Mathematics, Trinity College Dublin, Dublin 2, Ireland \\ e-mail: rharte@maths.tcd.ie \\ and AN-HYUN KIM \\ Department of Mathematics, Changwon National University, Changwon, Korea \\ e-mail: ahkim@changwon.ac.kr
}

(Received 19 November 2009; accepted 18 June 2010)

\begin{abstract}
The 'polaroid' property transfers from Banach algebra elements to their tensor product, and hence also to their induced multiplications on 'ultraprime' Banach bimodules.
\end{abstract}

2010 Mathematics Subject Classification. Primary 47B47; Secondary 47A10, 47A11.

1. Introduction. Recall that an element $T \in G$ of a complex Banach algebra $G$, with identity I and invertible group $G^{-1}$, is simply polar $([\mathbf{1}, \mathbf{3}, \mathbf{4}$, Definition 7.3.5]) iff there is $S \in G$ for which

$$
T-T S T=0=T S-S T
$$

the products

$$
T^{\bullet}=T S=S T, T^{\times}=S T S
$$

are uniquely determined and double commute with $T$. More generally $T \in G$ is polar iff $T^{n}$ is simply polar for some $n \in \mathbf{N}$, and quasi-polar iff ([3, 4, Definition 7.5.2]; cf. [8]) there is $E=E^{2}=I-E^{\prime} \in G$ for which

$$
T E=E T ; T E^{\prime} \in\left(E^{\prime} G E^{\prime}\right)^{-1} ; T E \in Q N(E G E) .
$$

Here

$$
Q N(G)=\left\{T \in G:\left\|T^{n}\right\|^{1 / n} \rightarrow 0(n \rightarrow \infty)\right\}=\left\{T \in G: I-\mathbf{C} T \subseteq G^{-1}\right\}
$$

are the quasi-nilpotent elements of $G$, and necessary and sufficient for $T \in G$ to be quasi-polar is that zero is at worst an isolated point of spectrum:

$$
0 \notin \operatorname{acc} \sigma(T) \subseteq \mathbf{C} .
$$


We recall $[\mathbf{1 , 6}]$ 'isoloid' and 'polaroid' elements:

Definition 1. $T \in G$ is said to be left (resp. right) isoloid if there is implication, for arbitrary $v \in \mathbf{C}$,

$$
T-v I \text { quasi-polar } \Longrightarrow T-v I \text { left (resp. right) zero divisor, }
$$

and polaroid if

$$
T-v I \text { quasi-polar } \Longrightarrow T-v I \text { polar, }
$$

In this paper we show that whenever $a \in A$ and $b \in B$ are polaroid then so is $T=$ $a \otimes b \in G=A \otimes B$, a uniformly cross-normed tensor product algebra, and hence also $T=L_{a} R_{b} \in G=B(M)$, induced 'elementary operators' on 'ultraprime' bimodules. We recall $([\mathbf{2}, \mathbf{4}$, Theorems 11.7.6 and 11.6.8]) a little bit of spectral theory,

$$
\sigma(a \otimes b)=\sigma(a) \sigma(b)=\sigma\left(L_{a} R_{b}\right),
$$

with an accompanying fragment of topology: if $K, H$ are compact subsets of $\mathbf{C}$ there is ([6, Theorem 6]) inclusion

$$
\operatorname{iso}(K \cdot H) \backslash\{0\} \subseteq \operatorname{iso}(K) \cdot \operatorname{iso}(H) \subseteq \text { iso }(K \cdot H) \cup\{0\}
$$

and

$$
\operatorname{iso}(K \cdot H) \subseteq \operatorname{iso}(K) \cdot H \cup K \cdot \operatorname{iso}(H) ;
$$

conversely,

$\operatorname{acc}(K) \cdot \operatorname{acc}(H) \subseteq \operatorname{acc}(K \cdot H) \subseteq \operatorname{acc}(K) \cdot H \cup K \cdot \operatorname{acc}(H) \subseteq \operatorname{acc}(K \cdot H) \cup\{0\}$.

As a supplement to (2.4) and (2.5),

THEOREM 2. If $K, H$ are compact subsets of $\mathbf{C}$ there is implication

$$
0 \in \text { (iso } K \cdot H) \backslash H \Longrightarrow 0 \in \text { iso } K,
$$

and

$$
0 \in \text { (iso } K \cdot H) \cap \text { acc } H \Longrightarrow K=\{0\} .
$$

Proof. If 0 is an isolated point of $K \cdot H$ then $0=\lambda \mu$ with $\mu \in H$ and $\lambda \in K$, and if $0 \notin H$ then necessarily $\lambda=0$. Now if $0 \in \operatorname{acc} K$ then there is $\left(\lambda_{n}\right)$ in $K$ with $0 \neq \lambda_{n} \rightarrow 0$ in which case $\mu \in H \Longrightarrow 0 \neq \lambda_{n} \mu \rightarrow 0$, contradicting the fact that 0 is isolated in $K \cdot H$. This gives (12); towards (13) suppose that $0 \neq \lambda \in K$ and $0 \neq \mu_{n} \rightarrow \mu$ in $H$ : then $0 \neq \lambda \mu_{n} \rightarrow 0$ in $K \cdot H$, again contradicting the status of 0 as an isolated point of $K \cdot H$

If $a \in A$ and $b \in B$ are left, or right, isoloid then so is $a \otimes b \in A \otimes B$ : this follows from Theorem 7 of [6], cf. [10], applied to the operators $L_{a}$ and $R_{b}$. The polaroid property also transfers:

Theorem 3. If $a \in A$ and $b \in B$ are polaroid then so is $T=a \otimes b \in G=A \otimes B$. 
Proof. If $0 \neq v \in$ iso $\sigma(a \otimes b)$ then by (9) there is $(\lambda, \mu) \in \mathbf{C}^{2}$ for which

$$
\lambda \in \text { iso } \sigma(a), \quad \mu \in \text { iso } \sigma(b), \quad \lambda \mu=v \text { : }
$$

then with $p=p^{2} \in A$ obtained from the analogue of (3) with $a-\lambda \in A$ in place of $T \in G$, and $q=q^{2} \in B$ doing the same job for $b-\mu \in B$ we have, with $p^{\prime}=1-p$, $q^{\prime}=1-q$ and $v=\lambda \mu$,

$$
\begin{aligned}
T= & a \otimes b-v(1 \otimes 1)=(a \otimes b-v(1 \otimes 1))\left(p^{\prime} \otimes q^{\prime}\right) \\
& +((a-\lambda) \otimes(b-\mu)+\lambda \otimes(b-\mu)+(a-\lambda) \otimes \mu)\left(p \otimes q+p^{\prime} \otimes q+p \otimes q^{\prime}\right) \\
= & ((a-\lambda) \otimes(b-\mu)+\lambda \otimes(b-\mu)+(a-\lambda) \otimes \mu)(p \otimes q) \\
& +((a-\lambda) \otimes b+\lambda \otimes(b-\mu))\left(p \otimes q^{\prime}\right)+(a \otimes(b-\mu)+(a-\lambda) \otimes \mu)\left(p^{\prime} \otimes q\right) \\
& +(a \otimes b-v(1 \otimes 1))\left(p^{\prime} \otimes q^{\prime}\right) .
\end{aligned}
$$

Now $T(p \otimes q)$ is the sum of three commuting nilpotents in $(p \otimes q) G(p \otimes q)$, each of $T\left(p \otimes q^{\prime}\right)$ and $T\left(p^{\prime} \otimes q\right)$ is the commuting sum of an invertible and a nilpotent, while finally the invertibility of $T\left(p^{\prime} \otimes q^{\prime}\right)$ in $\left(p^{\prime} \otimes q^{\prime}\right) G\left(p^{\prime} \otimes q^{\prime}\right)$ is (8), and $T \in G$ is therefore polar.

It remains to consider the case

$$
v=0 \in \text { iso } \sigma(a \otimes b) \subseteq \text { (iso } \sigma(a)) \sigma(b) \cup \sigma(a)(\text { iso } \sigma(b)) \text { : }
$$

necessarily $0 \in \sigma(a) \cup \sigma(b)$ and there are several possibilities. Note that there is implication

$$
a \in A \text { polar }, \quad b \in \text { B polar } \Longrightarrow a \otimes b \in A \otimes B \text { polar } .
$$

If $0 \in$ (iso $\sigma(a \otimes b)) \backslash \sigma(b)$ then $b \in B^{-1}$ and hence, by (12), $0 \in$ iso $\sigma(a)$. Thus 0 is a pole for $a \in A$ and an (honorary!) pole of $b \in B$. If $0 \in$ (iso $\sigma(a)) \cap(\operatorname{acc} \sigma(b))$ then necessarily, by (13), $\sigma(a)=\{0\}$ and hence also $\sigma(a \otimes b)=\{0\}$. Since $a \in A$ is polar and quasi-nilpotent it is also nilpotent, and hence also $a \otimes b$. If $0 \in($ iso $\sigma(a \otimes b)) \cap$ (iso $\sigma(b)$ ) then we again consider cases: either $0 \notin \sigma(a)$ in which case $a$ is invertible and $b$ is polar, or $0 \in \operatorname{acc} \sigma(a)$, in which case $b$ and hence also $a \otimes b$ are nilpotent, or finally $0 \in$ iso $\sigma(a)$, in which case both $a$ and $b$ are polar

The extension to multiplication operators is almost automatic:

COROLlary 4. If $a \in A$ and $b \in B$ are left isoloid, or polaroid, then so is $T=L_{a} R_{b} \in$ $G=B(M)$, for an ultraprime Banach $(A, B)$ bimodule $M$.

Proof. The prime condition [5],

$$
L_{a} R_{b}=0 \in B(M) \Longrightarrow 0 \in\{a, b\} \subseteq A \cup B,
$$

says that the 'elementary operators' induced by $A$ and $B$ on $M$ are just the tensor product of the algebras $L_{A} \subseteq B(M)$ and $R_{B} \subseteq B(M)$, and hence of the algebras $A$ and $B^{o p}$, obtained by reversing the multiplication in $B$, while the ultraprime condition,

$$
\left\|L_{a} R_{b}\right\|=\|a\|\|b\|
$$


ensures that the operator norm of $B(M)$ induces a uniform cross-norm on the tensor product

The Browder spectrum is given, with a little help from the punctured neighbourhood theorem, by

$$
\beta_{\text {ess }}(T)=\sigma_{\text {ess }}(T) \cup \operatorname{acc} \sigma(T)=\omega_{\text {ess }}(T) \cup \operatorname{acc} \sigma(T):
$$

in [6] this was written as $\omega_{\text {ess }}^{c o m m}(T)$. It is clear from (6) and the inclusion ([6, Theorem 3])

$$
\sigma_{\text {ess }}(a \otimes b) \subseteq \sigma_{\text {ess }}(a) \sigma(b) \cup \sigma(a) \sigma_{\text {ess }}(b)
$$

that

$$
\beta_{\text {ess }}(a \otimes b) \subseteq \beta_{\text {ess }}(a) \sigma(b) \cup \sigma(a) \beta_{\text {ess }}(b) \subseteq \beta_{\text {ess }}(a \otimes b) \cup\{0\} .
$$

The obstacle to the transfer of Browder's theorem lies in the slightly complicated form ([6, equation (6.6)]) of the Weyl spectrum of a tensor product. We begin by simplifying (21):

Theorem 5. If $a \in A=B(X)$ and $b \in B=B(Y)$ then

$$
\beta_{\text {ess }}(a \otimes b)=\beta_{\text {ess }}(a) \sigma(b) \cup \sigma(a) \beta_{\text {ess }}(b) .
$$

Proof. We recall ([6, Theorem 4]) the inclusion

$$
\left(a^{-1}(0) \otimes Y\right) \cup\left(X \otimes b^{-1}(0)\right) \subseteq(a \otimes b)^{-1}(0 \otimes 0),
$$

which ensures that, if both $X$ and $Y$ are infinite-dimensional, the operator $a \otimes b$ cannot have a non-trivial finite-dimensional null space: hence

$$
0 \in \sigma(a \otimes b) \Longrightarrow 0 \in \omega_{\text {ess }}(a \otimes b) \subseteq \beta_{\text {ess }}(a \otimes b) ;
$$

this with (20) gives (22)

If we also look at dual operators we can improve (6.3) to

$$
0 \in \sigma(a \otimes b) \Longrightarrow 0 \in \sigma_{\text {ess }}(a \otimes b) .
$$

Our observation now is that for any operators $a \in A$ and $b \in B$ for which 'Browder's theorem holds' simultaneously for $a, b$ and $a \otimes b$, the Weyl spectrum of $a \otimes b$ is comparatively simple:

THEOREM 6. If Browder's theorem holds for $a \in A=B(X)$ and $b \in B=B(Y)$ then the following are equivalent:

$$
\begin{gathered}
\omega_{e s s}(a \otimes b)=\omega_{e s s}(a) \sigma(b) \cup \sigma(a) \omega_{e s s}(b) . \\
\beta_{e s s}(a \otimes b)=\omega_{e s s}(a \otimes b) .
\end{gathered}
$$

Proof. If (26) holds then (cf. [10]) (27) follows from (24) and Browder's theorem for $a$ and $b$; conversely (27) and (22) give (26) 
Theorem 6 has been obtained for Hilbert spaces by Kubrusly and Duggal ([9, Proposition 7]). Kitson et al. [7] has a specific example in which the equivalent conditions of Theorem 6 both fail: with the forward and backward shifts $u$ and $v$ on $Y=\ell_{2}$, for which

$$
v u=1 \neq u v \in 1+\{c \in B(Y): \operatorname{dim} c(Y)<\infty\},
$$

take

$$
A=B(X), \quad X=Y \oplus Y, \quad a=(1-u v) \oplus\left(\frac{1}{2} u-1\right), \quad b=-(1-u v) \oplus\left(\frac{1}{2} v+1\right) .
$$

Acknowledgements. The authors wish to thank Slavisa Djordjevic, and the Mathematics faculty at Benemérita Universidad Autónoma de Puebla, for important conversations, and extraordinary hospitality, during this writing.

\section{REFERENCES}

1. B. P. Duggal, R. E. Harte and I. H. Jeon, Polaroid operators and Weyl's theorem, Proc. Amer. Math. Soc. 132 (2004), 1345-1349.

2. R. E. Harte, Tensor products, multiplication operators and the spectral mapping theorem, Proc. R. Irish Acad. 73(A) (1973), 285-302. $151-176$.

3. R. E. Harte, Fredholm, Weyl and Browder theory, Proc. R. Irish Acad. 85(A) (1986), 1988).

4. R. E. Harte, Invertibility and singularity for bounded linear operators (Dekker, New York,

5. R. E. Harte and C. Hernandez, On the Taylor spectrum of left-right multipliers, Proc. Amer. Math. Soc. 126 (1998), 103-118.

6. R. E. Harte and A.-H. Kim, Weyl's theorem, tensor products and multiplication operators, J. Math. Anal. Appl. 336 (2007), 1124-1131.

7. D. Kitson, R. E. Harte and C. Hernandez, Weyl's theorem and tensor products: A counterexample J. Math. Anal. Appl. (to appear).

8. J. J. Koliha, A generalized Drazin inverse, Glasgow Math. J. 38 (1996), 367-381.

9. C. S. Kubrusly and B. P. Duggal, On Weyl and Browder spectra of tensor products, Glasgow Math. J. 50 (2008), 289-302.

10. Y.-H. Song and A.-H. Kim, Weyl's theorem for tensor products, Glasgow Math. J. 46 (2004), 301-304. 